\title{
Incidental findings on hybrid SPECT-CT and PET- CT scanners: Is it time for new training and reporting guidelines?
}

\author{
Steven Port, $M D^{a}$ \\ a Aurora Cardiovascular Services, Aurora Health Care, Milwaukee, WI
}

Received Feb 24, 2018; accepted Feb 26, 2018

doi: $10.1007 / \mathrm{s} 12350-018-1257-0$

See related article, pp. 1688-1693

\section{ELECTRON BEAM CT WAS FIRST}

The first report of incidental findings on CT scans that had been acquired during the evaluation of subjects with known or suspected coronary heart disease came from Hunold et al in 2001. ${ }^{1}$ In that report, 1812 consecutive patients underwent electron beam CT scanning for detection and quantification of coronary artery calcium. All scans were assessed for noncoronary findings using only a tissue window. Extra-coronary findings were identified in $52 \%$ of the patients but included cardiac findings. The latter accounted for $71 \%$ of the noncoronary findings in those patients. Aortic "disease" was identified in $23 \%$ of the total group of which $90 \%$ included those with varying degrees of calcification, and $10 \%$ included aortic ectasia $(n=41)$ and aortic aneurysm $(n=11)$. One patient had an ascending aortic dissection that required surgery. Noncoronary, noncardiac, and nonvascular findings included two bronchial and one previously unknown but inoperable esophageal carcinoma detected $(0.16 \%$ of the total group). Numerous other noncardiovascular findings were noted, some of which led to additional testing including additional radiation. There was the usual potpourri of findings that one would expect in a population with a mean age of 59 , ranging up to 86 . No other life-saving findings were noted, but useful and

Reprint requests: Steven Port, MD, Aurora Health Care, Aurora Cardiovascular Services, Milwaukee, WI; sport@wi.rr.com

J Nucl Cardiol 2019;26:1694-6.

1071-3581/\$34.00

Copyright (C) 2018 American Society of Nuclear Cardiology. previously unknown diagnoses were made such as hiatus hernias that may have explained chest pain.

\section{LOW-DOSE, LOW-RESOLUTION SPECT-CT}

In 2006, Goetze et al reported on the abnormal findings detected on low-dose, low-resolution CT used for attenuation correction of SPECT studies. ${ }^{2}$ In that study, the CT scans were evaluated retrospectively in 200 patients, mean age 55, ranging from 17 to 87 . The CT scanner was a single-slice, nonspiral machine. The tube current was only $2.5 \mathrm{~mA}$ and slice thickness $10 \mathrm{~mm}$. No breath hold was employed due to the average 5 minute acquisition time. CT images were reviewed independently on the vendor-specific workstation by an expert in body CT. Scans were read in soft tissue, lung, and bone windows. They found 234 abnormalities in 119 of the 200 patients (59.5\%). Findings were categorized as major (25), minor (64), minimal (131), and equivocal (14). Examples of the "major" findings included coronary calcification, dilated ascending aorta, pericardial effusion, lung nodules, infiltrates, and masses, breast calcification, and a probable pericardial cyst. 15/25 major findings were previously unknown but 8 of those were coronary calcifications, 6 of whom had known coronary artery disease or some known coronary calcifications. Therefore, only $9 / 25$ major findings were previously unknown.

\section{HIGH-DOSE, HIGH-RESOLUTION SPECT-CT}

In 2009, Husmann et al documented the prevalence of noncardiac findings in 582 consecutive patients who underwent SPECT-CT imaging for known or suspected coronary artery disease. ${ }^{3}$ In contrast to the study of Goetze et al., the CT scanner in this study was a 64-slice machine. Images were acquired at $2.5 \mathrm{~mm}$ slice 
thickness, $120 \mathrm{kEV}$ and $200 \mathrm{~mA}$ using a $50-\mathrm{cm}$ field of view, and subsequently analyzed on a dedicated CT workstation (GE AW4.4). All CT images were read by a board-certified radiologist using standard soft tissue, lung, and bone windows. The mean age of the group was 64 ranging up to 89 . A total of $400(68.7 \%)$ of the patients had a noncardiac finding including 226 findings in 196 patients $(33.6 \%)$ who were deemed to have findings of potential clinical relevance. Of those 196 patients, 156 had pulmonary nodules requiring followup (noncalcified, $>10 \mathrm{~mm}$ or $<10 \mathrm{~mm}$ with irregular margins). Subsequent follow-up confirmed 3 newly diagnosed bronchial carcinomas $(0.5 \%$ of the total group). However, over half the group with possibly relevant findings were lost to follow-up. Aortic aneurysms were identified in 5 patients and aortic dissection in 4 patients.

\section{LOW-DOSE, HIGH-RESOLUTION PET-CT}

In 2017, Kan et al reported on the incidence of extra-cardiac findings on ${ }^{13} \mathrm{NH}_{3}$ MPI. ${ }^{4}$ The group consisted of 1397 consecutive patients between 2013 and 2016 being studied for possible myocardial ischemia. The mean age was 66.8 , ranging up to 90 . The transmission scans were acquired on a 16-slice CT scanner using $130 \mathrm{kVp}$ and $25 \mathrm{mAs}$ without breath hold and were reconstructed with a large field-of-view with $3 \mathrm{~mm}$ slice thickness. Interpretations were performed by experienced nuclear medicine physicians. There were a total of 775 incidental noncardiac findings in 717 (51\%) patients, of which 515 were deemed clinically irrelevant and 260 possible relevant. Of the 260,115 were deemed potentially clinically relevant and 145 were diagnosed with findings that could have explained the symptom that led to the perfusion imaging. There were lung nodules in 49/115 which led to a diagnosis of primary or metastatic carcinoma in 4 patients. Lymph nodes $>10$ mm were identified in 33/115 (29\%). Two of those had lymphoma, one had sarcoidosis, one had metastatic gastric carcinoma, and one had metastasis from an unknown primary. In the group with findings that could have explained chest pain symptoms, there were hiatus hernias in 86 patients $(59 \%)$, gallstones, in $36 \%$ and kidney stones in $5 \%$.

\section{CARDIOLOGY VS. RADIOLOGY}

The above discussion brings us to the latest study by He et al in which consecutive SPECT-CT MPI studies over an 8-month period were analyzed for noncardiac findings. ${ }^{5}$ Both 16- and 64-slice CT scanners were used with $120 \mathrm{KeV}$ and $30-60 \mathrm{~mA}$. Images were acquired with 3.8 (PET-CT) or $5 \mathrm{~mm}$ (SPECT-CT) slices. What makes this study different from earlier studies cited above is a comparison between cardiologist and radiologist interpretations of the extra-cardiac findings. As in the previous reports, there were many incidental findings with radiology identifying them in $41 \%$ and cardiology identifying them in $30 \%$ of the cohort. Substantial under-reporting of aortic aneurysms, hiatus hernias, and lung masses was noted in the cardiologists' interpretations compared with the radiologist. Lung nodules and pleural plaques were over-called by cardiology.

Several methodological issues are present in this study primarily because it was not prospectively designed to address the differences between cardiologists and radiologists in their ability to detect noncardiac findings on the CT portion of the hybrid study. The study was a retrospective review designed as a departmental quality-improvement project. No special training was offered to the cardiologists. There was no a priori agreement on what constituted an aortic aneurysm, and the radiologist used a maximally sensitive threshold of $3.5 \mathrm{~cm}$ for the definition of an aortic aneurysm. The cardiologists used standard nuclear cardiology interpretive software to review the CT data (AutoQuant for SPECT or Emory Toolbox for PET) with the option to use the same software used by the radiologist. It is not clear if or how often the cardiologists availed themselves of that option. The radiologist used dedicated CT image review software for all cases. The radiologist identified 124 aortic aneurysms, while cardiologists identified only 43. Such a huge discrepancy could only occur in the absence of a prospectively agreed upon definition of an aneurysm. By design, the said study certainly cannot be considered definitive in respect to the comparison between cardiology and radiology in the detection of incidental CT findings on hybrid MPI studies. Perhaps, a prospective study addressing the same question would be appropriate, especially if the playing field was the same for all participants. However, the paper is provocative and timely as hybrid systems continue to percolate into the nuclear cardiology imaging world.

\section{MAGNITUDE OF THE PROBLEM}

In all the studies cited herein, the fraction of incidental findings that were previously unknown was as high as $60 \%$. However, the number of incidental findings that led to potentially treatment-altering diagnoses was low with the identification of previously unknown and treatable malignancies no greater than $0.5 \%$. A number of noncalcified lung nodules requiring follow-up are found in all studies. Rare, previously unknown aortic aneurysms and aortic dissections have been found. Unsuspected sarcoidosis was reported in 
one patient. An occasional lymphoma has been newly diagnosed. In addition to the rare, potentially lifethreatening diagnoses, other clinically important findings arise somewhat more commonly such as pneumonia, pulmonary fibrosis, and diagnoses that might explain chest pain such as a hiatus hernia. Despite the inclusion of coronary artery calcification as an incidental finding in the early papers on this subject, the presence of coronary calcification should be considered integral to, not incidental to the MPI findings.

There is, therefore, no doubt that potentially important, incidental findings will occasionally be found when the CT datasets of the hybrid SPECT-CT and PET-CT MPI studies are carefully reviewed in both tissue and lung windows. That appears to be true with CT scanners ranging from single slice to 64-slice and from 2.5 to $200 \mathrm{~mA}$ acquisitions. Good care and medicolegal liability will dictate that cardiologists and nuclear medicine physicians will have to consider including review of the CT data as an integral part of the interpretation and reporting of MPI studies that are performed on hybrid systems. But are cardiologists ready for that challenge?

\section{NEXT STEPS: TRAINING AND LAB ACCREDITATION}

The training guideline for nuclear medicine residents was modified in 2007 when primary nuclear medicine residency training was lengthened from 2 to 3 years. with the specific mandate of 6 months of training in cross-sectional anatomic imaging. ${ }^{6}$ As of COCATS 4 (2015), no specific recommendation has been advanced that would encourage or mandate training in chest CT for cardiologists. Nor has the CBNC required such training to become board certified in nuclear cardiology. And, in fairness to cardiologists, the majority of the existing SPECT systems are not hybrid systems. and many new systems, including advanced CZT systems, are not hybrid systems. Therefore, at this point time, it would seem unnecessary to burden all nuclear cardiology trainees with formal training in chest CT. Even training guidelines for multimodality imaging have yet to specifically address the issue of chest CT as part of training for nuclear cardiology and/or cardiac CT.

However, in the near future, it may become necessary for nuclear cardiology laboratories to demonstrate training of their readers in the interpretation of chest CT if a lab requests accreditation for hybrid SPECT/CT or PET/CT systems. Alternatively, a lab could provide independent review of all hybrid CT data by a radiologist trained in chest CT and subsequently produce a "hybrid"' report. Creating a single "hybrid" report from different providers in an electronic health record may not be as easy as it sounds and may require input from the EHR vendor and from local IT services. It certainly has not been an easy task in my environment.

The paper by He et al in this issue of the Journal is almost certainly the harbinger of new guidelines for the interpretation of MPI studies using hybrid machines. If they have not already done so, cardiologists and nuclear cardiology laboratories should start preparing.

\section{Disclosure}

The author declares that there is no conflict of interest to disclose.

\section{References}

1. Hunold P, Schmermund A, Seibel RM, Gronemeyer DH, Erbel R. Prevalence and clinical significance of accidental findings in electron-beam tomographic scans for coronary artery calcification. Eur Heart J. 2001;22:1748-58.

2. Goetze S, Pannu HK, Wahl RL. Clinically significant abnormal findings on the "Nondiagnostic" CT portion of low-amperage-CT attenuation-corrected myocardial perfusion SPECT/CT studies. J Nucl Med. 2006;47:1312-8.

3. Husmann L, Tatsugami F, Aepli U, Herzog BA, Valenta I, VeitHaibach P, Buechel RR, Pazhenkottil AP, Gaemperli O, Burkhard N, Wyss CA, Kaufmann PA. Prevalence of noncardiac findings on low dose 64-slice computed tomography used for attenuation correction in myocardial perfusion imaging with SPECT. Int J Cardiovasc Imaging. 2009;25:859-65.

4. Kan H, van der Zant FM, Wondergem M, Knol RJJ. Incidental extra-cardiac findings on ${ }^{13} \mathrm{~N}$-ammonia myocardial perfusion PET/ CT. J Nucl Cardiol. 2017;24:1860-8.

5. He BJ, Malm BJ, Carino M, Sadeghi MM. Prevalence and variability in reporting of clinically actionable incidental findings on attenuation-correction CT scans in a veteran population. J Nucl Cardiol. 2018. https://doi.org/10.1007/s12350-018-1232-9.

6. Delbeke D, Royal HD, Frey KA, Graham MM, Segall GM. SNMMI/ABNM joint position statement on optimizing training in nuclear medicine in the era of hybrid imaging. J Nucl Med. 2012;53:1490-4. 